\title{
Mörderische Überstunden
}

\begin{abstract}
Die Whitehall-II-Studie untersucht Auswirkungen verschiedener Aspekte der Arbeitswelt auf die Gesundheit der Berufstätigen. Probanden sind ca. 6000 britische Beschäftigte des öffentlichen Dienstes. Die neuesten Ergebnisse von Whitehall II zeigen, dass regelmäßige Überstunden das Risiko, an KHK zu erkranken, drastisch erhöhen.
\end{abstract}

- Bei Beginn der Studie (1991 bis 1994) waren die Probanden (Alter 39 bis 61 Jahre) herzgesund. Im Zeitraum von elf Jahren erlitten 369 Personen entweder einen tödlichen oder nicht tödlichen Infarkt oder erkrankten an Angina pectoris.

Mit den Cox-proportionalen Hazardmodellen wurde errechnet, dass Beschäftigte, die pro Tag drei bis vier
Überstunden machten, im Vergleich zu denen, die keine machten, ein um $60 \%$ (HR 1,60; 95\%-KI: 1,15-2,23) erhöhtes Risiko hatten, an einer KHK zu erkranken. Wurden andere, nicht arbeitsbedingte KHK-Risikofaktoren herausgerechnet, blieb für die Überstunden immer noch eine HR von 1,56 (95\%-KI: 1,11-2,19).

\section{Kommentar}

Überstunden sind demnach unabhängig von den konventionellen Risikofaktoren ein eigenständiger Risikofaktor für Herzerkrankungen. Zum Teil lässt sich dies wohl aus der Persönlichkeitsstruktur der Probanden erklären: Überstunden machen vor allem Typ-A-Persönlichkeiten, die immer unter „Dampf “ stehen und häufiger unter Bluthochdruck leiden. Wer viele Überstunden leistet, hat auch weniger Zeit, sich um gesunde Ernährung,

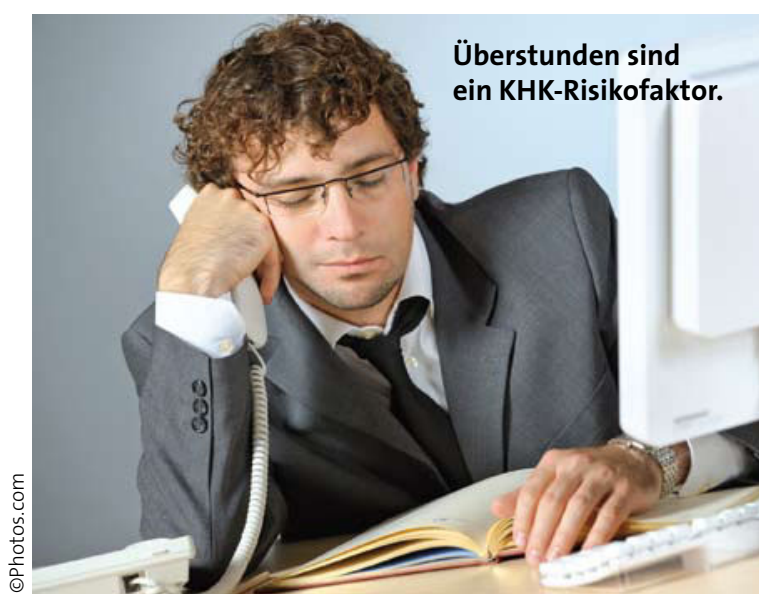

ausgleichende Bewegung und regelmäßige Arztbesuche zu kümmern, und leidet häufiger an Schlafmangel, Angststörungen und Depressionen. K. MALBERG .

- M. Virtanen et al.

Overtime work and incident coronary heart disease: the Whitehall II prospective cohort study. Eur Heart J. Published online May 11, 2010 